vol. $37-n^{\circ} 1$ et 2 | 2021

Travail et migrations

\title{
Lendaro Annalisa, Rodier Claire, Vertongen Youri Lou (dir.), La Crise de l'accueil. Frontières, droits, résistances
}

\section{Ralph Schor}

\section{(2) OpenEdition}

12 Journals

Édition électronique

URL : https://journals.openedition.org/remi/18764

DOI : $10.4000 /$ remi. 18764

ISSN : $1777-5418$

Éditeur

Université de Poitiers

Édition imprimée

Pagination : 321-323

ISBN : 979-10-90426-69-6

ISSN : 0765-0752

\section{Référence électronique}

Ralph Schor, « Lendaro Annalisa, Rodier Claire, Vertongen Youri Lou (dir.), La Crise de l'accueil.

Frontières, droits, résistances ", Revue européenne des migrations internationales [En ligne], vol. 37 - n 1 et 2 | 2021, mis en ligne le 25 novembre 2021, consulté le 16 mai 2022. URL : http://

journals.openedition.org/remi/18764; DOI : https://doi.org/10.4000/remi.18764

Ce document a été généré automatiquement le 16 mai 2022.

(c) Université de Poitiers 


\title{
Lendaro Annalisa, Rodier Claire, Vertongen Youri Lou (dir.), La Crise de l'accueil. Frontières, droits, résistances
}

\author{
Ralph Schor
}

\section{RÉFÉRENCE}

Lendaro, Annalisa (dir.), Rodier, Claire (dir.), Vertongen, Youri Lou (dir.), La Crise de l'accueil. Frontières, droits, résistances. - Paris : La Découverte, 2019. - 316 p. ISBN :

978-2-34804-284-3

1 Un important ouvrage collectif, rédigé par des politistes, des juristes, des géographes, des sociologues et des anthropologues, a été consacré à ce qui est communément appelé la crise des réfugiés ou, plus exactement, la crise de l'accueil qui se développe depuis 2015. Les épisodes dramatiques de cette crise sont suivis de multiples conséquences : nombreuses morts sur les routes terrestres de la migration et surtout durant la traversée de la Méditerranée, chaos dans la gestion politique et juridique du drame, durcissement des règles de l'asile, externalisation de la surveillance des frontières et sous-traitance de cette tâche à des pays non européens, inégalité du choc ressenti par les pays concernés - l'Italie et la Grèce se retrouvent en première ligne naissance de réseaux de solidarité spontanée avec les migrants. Toutes ces questions font l'objet de mises au point précises et richement documentées.

2 L'histoire de la crise de 2015 et de ses signes avant-coureurs caractérise les grandes étapes du phénomène et les réactions des États. L'étude fait ressortir les dissensions survenues entre la Commission européenne et les États membres, puis entre ces derniers eux-mêmes. Les idéologies et les intérêts se révèlent en effet divergents. Dans certains pays, l'extrême droite exerce une forte pression xénophobe et va jusqu'à 
accuser les ONG de complicité avec les passeurs. La générosité initiale de l'Allemagne à l'égard des demandeurs d'asile est parfois considérée comme intéressée, car le gouvernement de Berlin, obéissant au patronat, aurait voulu en fait introduire de la main-d'œuvre pour compenser un important déficit démographique. D'une manière générale, les États sont accusés d'afficher un respect apparent des droits de réfugiés et, en réalité, de considérer ceux-ci comme des migrants économiques déguisés qu'il faut écarter au maximum et sélectionner selon des critères de plus en plus drastiques. Plusieurs auteurs pensent que "l'invasion" représente un fantasme et qu'en se barricadant au mépris du droit, l'Europe renie ses valeurs fondamentales, assises de son identité. Considérer le réfugié comme un menteur potentiel revient à ignorer la réalité, souvent indicible, de l'exil et à ériger en principe la subjectivité des fonctionnaires chargés d'instruire les dossiers d'admission à l'asile. Les catégorisations juridiques et politiques, censées codifier la situation des migrants, se révèlent en fait très contingentes: avant l'augmentation du chômage, depuis 1974, la France s'ouvrait largement aux migrants économiques ; les associations qui aident les ayants droit dans leurs démarches utilisent des critères variables selon les époques; par opposition aux "réfugiés potentiellement fraudeurs » d'aujourd'hui, les réfugiés des années 1990, Vietnamiens, Chiliens, Argentins, faisaient figure de héros.

3 Les auteurs illustrent leur propos de manière concrète grâce à une série de monographies. Ainsi la Bulgarie, théoriquement pays de transit pour les migrants, est devenue une sentinelle postée sur la frontière extérieure de l'Europe, sentinelle chargée de décourager les exilés par la multiplication des mesures coercitives. Le Royaume-Uni a adopté depuis 1990 huit réformes majeures qui ont réduit les droits des demandeurs d'asile. Le Brexit a lui-même été présenté comme un moyen de reprendre le contrôle des frontières britanniques. Dans ce pays, les administrations, les banques, les logeurs sont devenus gestionnaires de nouvelles frontières quotidiennes, car ils doivent, sous peine d'amendes ou d'emprisonnement, vérifier la validité des titres de séjour. Dans le même temps, la frontière géographique a été délocalisée à Calais et à Dunkerque. Depuis 2011, l'afflux des migrants à Vintimille et à Menton a été dramatisé pour permettre aux forces de l'ordre de demander des moyens supplémentaires et d'effectuer des contrôles qui ne respectent pas toujours la lettre de la loi. De Sangatte à la Jungle de Calais, véritable quartier à la fois nouveau et précaire, l'étude montre la transformation de la région en un limes britannique, l'intervention plus ou moins forte de l'État français, l'action de bénévoles associatifs parfois en situation de rivalité, s'accusant réciproquement d'amateurisme ou de professionnalisme excessif, de collusion avec les pouvoirs publics ou de contestation dogmatique. À Melilla, seule frontière terrestre entre l'Europe et l'Afrique, les autorités ont soigneusement enclos le territoire, ce qui accroît les risques lors des franchissements clandestins. Les morts sont cachés au maximum et ne donnent pas lieu à un dénombrement officiel, mais, depuis la fin des années 1980, le chiffre pourrait dépasser les 20 000. En Allemagne, le déferlement des migrants a suscité à la fois de violentes critiques dans les milieux les plus conservateurs et l'apparition d'une véritable culture de l'accueil chez de nombreux citoyens ancrés à gauche et, de plus en plus, au centre de la société, avec une surreprésentation des femmes et des diplômés. Cet engagement humanitaire entraîne deux interprétations différentes: d'une part, ce serait une noble pratique philanthropique favorisant la compréhension et l'intégration des exilés, d'autre part ce serait une attitude paternaliste réduisant les demandeurs d'asile à un groupe passif et dépendant de la charité. Le gouvernement hongrois traite avec brutalité les migrants 
présentés comme des terroristes menaçant l'existence même de la nation. Cependant, dans les espaces-frontières, comme à Szeged et à Pecs, des comités solidaires des exilés se sont créés. Tout en se disant apolitiques, ils représentent une forme d'opposition au pouvoir de Victor Orban et se placent dans le sillage d'autres luttes menées en Hongrie en faveur de groupes marginalisés comme les Roms. Les hotspots, ceux de Lampedusa et de Lesbos, comme les huit autres créés en Italie et en Grèce, servent essentiellement à identifier, classer et surtout bloquer les nouveaux arrivants. Ces points, dotés au départ d'objectifs humanitaires et sécuritaires, ont mis l'accent sur leur deuxième mission. On y retrouve les défauts enregistrés dans les centres antérieurs : surpopulation, manque d'informations et d'interprètes, privations illégales de libertés.

Dans une conclusion très ferme, Danièle Lochak souligne la contradiction qui oppose le principe de l'universalité des droits de l'homme et la reconnaissance de la souveraineté des États qui traitent les étrangers selon leurs propres règles. Or les règles juridiques internationales garantissent de nombreux droits fréquemment violés : la faculté de quitter son propre pays, le non-refoulement consacré par la Convention de Genève, l'asile, l'interdiction des traitements inhumains et dégradants, la liberté et la sûreté, l'interdiction de la détention arbitraire, le secours en mer obligatoire et, en définitive, le droit à la vie. Les entorses à ces droits sont reconnues, mais la Cour européenne des droits de l'homme admet l'intérêt prééminent des États à contrôler les flux migratoires. Une grande marge d'impunité est donc possible, surtout quand les États européens confient à des pays tiers le soin de dissuader les migrants d'arriver sur le vieux continent. Le droit montre ainsi ses limites et sa marge d'impuissance. Il en va de même pour les autorités morales : le Saint-Siège reconnaît aux États la faculté de fermer leurs frontières, sauf au détriment des réfugiés menacés dans leur vie. Jusqu'à quand les défenseurs des droits de l'homme en seront-ils réduits à clamare in deserto?

\section{AUTEURS}

\section{RALPH SCHOR}

Historien, Professeur émérite

Université Côte-d'Azur/CMMC 